\title{
Expérimentations italiennes
}

\section{Francesca Bertè}

\section{OpenEdition}

\section{Journals}

Édition électronique

URL : http://journals.openedition.org/ries/3402

DOI : 10.4000/ries.3402

ISSN : 2261-4265

\section{Éditeur}

Centre international d'études pédagogiques

\section{Édition imprimée}

Date de publication : 1 mars 1996

Pagination : 113-118

ISSN : 1254-4590

\section{Référence électronique}

Francesca Bertè, "Expérimentations italiennes », Revue internationale d'éducation de Sèvres [En ligne],

09 | 1996, mis en ligne le 19 août 2013, consulté le 23 mars 2021. URL : http://

journals.openedition.org/ries/3402 ; DOI : https://doi.org/10.4000/ries.3402

Ce document a été généré automatiquement le 23 mars 2021.

() Tous droits réservés 


\title{
Expérimentations italiennes
}

\author{
Francesca Bertè
}

1 En Italie, dès le milieu des années soixante-dix, introduire une langue étrangère à l'école primaire est apparu comme une nécessité qui n'a cessé de s'affirmer. Le psycholinguiste qui a ouvert le débat sur l'opportunité d'une approche précoce des langues étrangères, Renzo Titone, affirme que trois grands axes déterminent cette opportunité :

- des raisons d'ordre neurologique: la plasticité neuro-cérébrale propre à l'âge infantile facilite l'acquisition d'une langue étrangère ;

- des raisons d'ordre psycho-évolutive : la facilité de l'apprentissage est également due aux caractéristiques psychologiques de l'enfant; la spontanéité et l'absence d'inhibitions ont dans ce cas une incidence très favorable ;

- des raisons d'ordre social: la confrontation, à travers la langue, à une autre culture, prédispose à une plus grande ouverture tout en étant une incitation à dépasser des attitudes égocentriques.

\section{Premières expériences : le projet ILSSE'1 (1977-1985)}

2 Les premières expériences d'enseignement d'une langue étrangère à l'école élémentaire ont débuté une dizaine d'années avant que ne voient le jour les programmes de 1985, dans les situations et les modalités les plus variées. Elles ont tout de suite mis en évidence la nécessité de définir le statut à donner à la langue étrangère en tant que discipline. Le texte du programme résume les résultats d'un débat né d'expériences mises en parallèle, et en tout premier lieu le Progretto ILSSE, qui avait commencé en 1977 et avait abouti à la publication des résultats en 1985, précisément.

3 À propos de cette expérimentation, on a conclu qu'« une grande attention a été portée à la problématique développée par la recherche linguistique et la pédagogie de l'enseignement des langues. La méthode fonctionnelle, qui fait suite chronologiquement à l'approche structurale et en représente un développement en direction de l'approche communicative s'est révélée la plus adéquate pour l'élaboration d'une didactique spécifique visant les élèves de l'école primaire. Les travaux effectués 
par les experts du Conseil de L'Europe dans les années soixante-dix, Un Niveau-seuil et le Threshold-Level pour l'enseignement et l'apprentissage du français et de l'anglais, sont devenus un point de référence constant. Une recherche spécifique est cependant nécessaire pour mettre en évidence les fonctions linguistiques développées prioritairement par les enfants de sept à dix ans. On a alors fait une enquête préliminaire dans quelques écoles romaines, ce qui a permis l'émergence de ces fonctions linguistiques, plus particulièrement la fonction sociale et la fonction émotive, avec une nette avance sur les fonctions de type heuristique ou logique. »

De l'étude de la méthodologie fonctionnelle en relation avec les exigences particulières d'une adaptation à la réalité spécifique de l'école primaire, on est arrivé à la définition de trois aspects essentiels à prendre en compte au moment de la formulation d'un plan de travail et de la préparation du matériel didactique. On peut de façon synthétique définir ces trois aspects en ayant bien présent à l'esprit qu'ils sont interdépendants :

- aspect génératif, dans le sens où la langue doit pouvoir être intériorisée et permettre de la façon où elle est présentée de générer le plus grand nombre possible d'expressions correctes ;

- aspect interdisciplinaire, l'interdisciplinarité étant une exigence épistémologique et didactique fondamentale au niveau de l'école élémentaire ;

- aspect multisensoriel, dans la mesure où l'enfant communique également par le mouvement, le jeu et la manipulation des objets.

5 Huit années d'expérimentation rigoureuse ont confirmé une hypothèse fondamentale : à l'école primaire la langue étrangère assume un rôle particulier qui relègue au second plan l'aspect utilitaire-instrumental, après un aspect de formation dans l'apprentissage, formation dans toute l'acception du terme. Le choix initial de confier l'enseignement de la langue étrangère à un maître non "spécialiste » a eu pour but de favoriser la recherche d'une méthodologie appropriée afin de l'intégrer, de la manière la plus harmonieuse, au programme de chaque classe expérimentale. Les itinéraires didactiques ont été élaborés durant les premières années par les maîtres expérimentateurs assistés de «tuteurs ». On retrouve dans les premières unités didactiques, construites par les équipes de coordination, une adhésion à la réalité enfantine et une progression en rapport avec le développement psychologique de l'élève plutôt qu'une progression sur le plan linguistique de type morphologique et structural.

\section{Les programmes de 1985}

6 Avec les programmes de 1985, la langue étrangère a été introduite en tant que discipline, participant d'une «vision globale de l'éducation linguistique». L'introduction à la partie disciplinaire du programme stipule que "L'éducation linguistique, dans une époque d'intenses communications et dans la perspective d'un processus croissant d'intégration dans la Communauté européenne ne peut se faire sans l'approche d'une langue étrangère. »

7 Le texte du programme de langue étrangère, eu égard à ces prémices, légitime une interprétation se fondant sur les bases épistémologiques du programme de langue italienne. C'est dans ce sens que l'on peut déterminer les objectifs que l'on se fixe quant à l'introduction de la langue étrangère. Celle-ci tient compte du cadre conceptuel déterminé dans les définitions de l'acquisition de la langue dans le programme de 
langue italienne. Les trois objectifs indiqués mettent l'accent sur l'aspect formatif de la seconde langue et et en soulignent les trois aspects fondamentaux.

Le premier objectif poursuivi par l'enseignement de la langue consiste à «favoriser le développement cognitif, en offrant un autre mode d'organisation des connaissances ", se posant ainsi comme instrument-unifiant de l'apprentissage. En effet, avant même d'être un instrument de communication, la langue est instrument de la pensée. Pour cette raison, le premier objectif insiste de façon éclairante sur l'aspect cognitif : la rencontre avec une autre langue correspond à une autre façon d'organiser la réalité.

9 La première définition de la langue donnée dans le programme de langue italienne est claire sur ce point: «La langue est instrument de la pensée, non seulement parce qu'elle la traduit en paroles (en permettant à l'individu de parler avec lui-même, c'està-dire de raisonner) mais aussi parce qu'elle sollicite et facilite le développement des processus mentaux qui organisent, de diverses manières, les données de l'expérience. »

10 Le deuxième objectif concerne l'aspect communicatif-instrumental. Il consiste à « permettre à l'enfant de communiquer avec d'autres au moyen d'une langue différente de la sienne ». On trouve ici un rappel de la seconde définition donnée à la langue qui permet "d'établir un rapport social ", "de communiquer avec d'autres " et où elle apparaît ainsi comme un moyen d'action. Pour atteindre cet objectif, il apparait nécessaire d'apporter une attention toute particulière au choix méthodologique. Pour développer des capacités de communication, il est indispensable de privilégier la langue orale avec toutes les implications didactiques que cela implique.

11 Le troisième objectif se propose "d'amener l'élève, au moyen de la langue, à comprendre d'autres cultures et d'autres peuples ", objectif qui n'est pas sans rapport avec la cinquième définition donnée à la langue. Celle-ci « est un objet culturel qui a pour dimension celle du temps historique, de l'espace géographique, de l'épaisseur sociale », d'où la nécessité de ne pas isoler l'enseignement de la seconde langue mais de l'intégrer à d'autres enseignements. Comprendre «d'autres cultures et d'autres peuples » favorise notamment une attitude de respect et développe l'intérêt pour des réalités culturelles différentes. Les définitions données à la langue ainsi que la détermination des objectifs donnent une vision globale de ce qu'est l'éducation pour accéder à la langue. En ce qui concerne l'école élémentaire, les études les plus récentes dans ce domaine de la pédagogie soulignent le lien entre les objectifs et l'approche méthodologique. Elles permettent d'appréhender le lien étroit entre la langue maternelle et la langue étrangère appartenant à un seul et unique domaine du point de vue de l'éducation.

12 L'approche communicative, de par l'attention qu'elle porte à la langue orale et au rapport existant entre la langue orale et la langue écrite, s'avère un leitmotiv méthodologique assurant une cohésion entre ces deux codes dans l'apprentissage des langues. En ce qui concerne la langue maternelle, il faut souligner «l'importance de l'attitude d'écoute, à ne pas confondre avec un comportement de réception passive, mais bien comme une capacité de compréhension et d'interprétation des messages ». L'enseignant doit être amené à savoir utiliser et «à favoriser des situations de communication ", comportement apparaissant comme fondamental du point de vue de la didactique des langues étrangères. La priorité accordée à la langue orale relève essentiellement de deux aspects, celui de l'interaction linguistique qui a normalement lieu à l'oral, et un autre aspect qui relève plus particulièrement de la didactique. Il est nécessaire de ne présenter à l'élève, au moins au début, sous forme écrite, que des 
structures et des mots déjà intériorisés au niveau de l'oral. Cela s'avère indispensable si l'on veut éviter des interférences sur le plan phonétique.

Un autre aspect fondamental dans le texte du programme de langues étrangères concerne l'approche ludique. On y trouve des indications particulièrement détaillées :

« Dès le début, on utilisera des tableaux, des dessins, des masques, des poupées et

des marionnettes, on organisera des jeux individuels et collectifs. »

14 Le jeu dans l'enseignement de la langue étrangère n'est pas seulement un expédient didactique. S'il est vrai qu'apprendre en se divertissant est plus agréable, ce n'est pas seulement pour cette raison que la pédagogie de la langue étrangère est centrée sur le jeu. Une raison bien plus profonde confère à l'approche ludique une valeur significative. Si l'on considère que l'acquisition d'une compétence communicative est un objectif fondamental, il est nécessaire de créer dans la classe des situations de communication authentique. Mais comment est-il possible de créer dans un contexte monolingue italien des situations authentiques d'un point de vue communicatif, en langue étrangère ? Le jeu, seul, est en mesure de créer cette situation. Les enfants sont disposés à entrer dans un autre univers à condition qu'ils y soient invités et sollicités en stimulant leur imagination: "Faisons mine de, imaginons que...». Dans le jeu, l'imaginaire devient authentique, la communication se réalise. Le jeu est donc un outil d'approche, le point de départ d'un itinéraire didactique complexe, à programmer de façon spécifique et en corrélation avec les autres disciplines de la classe.

\section{La réalisation des programmes : formation des enseignants et organisation}

15 Avec la réforme relative à l'organisation modulaire ${ }^{2}$, une importante innovation a vu le jour dans l'école italienne. Il n'y a plus un enseignant unique par classe mais trois enseignants opèrent sur deux classes. Nous sommes là dans une organisation modulaire où chaque maître se spécialise dans un ou plusieurs secteurs disciplinaires. C'est dans ce cadre qu'intervient l'enseignant de langue étrangère. Après avoir passé un examen, celui qui possède une certaine connaissance d'une langue étrangère peut suivre des cours de formation. Ces cours sont différenciés selon les degrés de compétence. Il existe des cours de cent, cent cinquante, trois cents ou cinq cents heures. La formation touche trois aspects: la linguistique, la pédagogie de l'enseignement de la langue et l'interculturel. Au terme de cette formation, l'enseignant peut intervenir selon les deux cas de figures prévus par la loi.

16 L'enseignant est « spécialiste »: dans ce cas, il pratique seulement l'enseignement de la langue étrangère et opère au niveau de plusieurs classes. Cette façon de procéder permet de toucher un plus grand nombre d'élèves et représente la solution à adopter en priorité dans cette phase transitoire.

17 L'enseignant est "spécialisé » : dans ce cas, la langue étrangère est enseignée par l'un des enseignants du module. De cette façon, il est possible de réaliser une intégration complète et fonctionnelle de la langue étrangère dans le programme. C'est la solution optimale tant qu'il n'y aura pas suffisamment d'enseignants préparés à cet enseignement.

18 En ce qui concerne le choix de la langue à enseigner, c'est le principe du pluralisme linguistique qui s'est affirmé. A l'école primaire italienne, on peut enseigner quatre 
langues européennes : l'anglais, le français, l'allemand et l'espagnol. Un sondage très précis portant sur la demande relative à ces quatre langues a été effectué au printemps 1992. Il entendait créer des conditions favorables au développement d'une action tenant compte des instances éducatives, culturelles et sociales des diverses réalités territoriales mais aussi des conditions d'une mise en œuvre de cette action dans les seuls contextes scolaires. Les classes ayant exprimé leur propre orientation quant aux quatre langues prévues par la réglementation étaient au nombre de 106947.

En ce qui concerne l'articulation de la demande, l'enquête sur les attentes et les orientations des milieux scolaires a mis en évidence trois aspects fondamentaux. À savoir, une très nette préférence pour l'anglais, en accord avec la réalité des autres pays européens ; une attention significative au français ; une demande plus modérée en ce qui concerne l'espagnol et l'allemand.

\section{L'anglais}

82,07 \% des classes ont choisi la langue anglaise. Le pourcentage baisse légèrement au nord et s'avère légèrement supérieur dans le centre de l'Italie. Le phénomène était préoccupant au départ car l'on craignait que le fait de proposer l'enseignement d'une langue autre que l'anglais n'amène une sérieuse protestation des parents. Cependant un travail intense de persuasion sur le terrain, dans le but de faire prendre conscience des objectifs de formation de la langue étrangère à l'école élémentaire a aidé à faire accepter le pluralisme linguistique. D'un point de vue quantitatif, la prédominance des classes d'anglais n'a pas été un obstacle à la présence des autres langues, garantie de dialogue et d'ouverture sur d'autres cultures.

\section{Le français}

$21 \quad 13,5 \%$ des classes ont choisi le français. Ce pourcentage tend à augmenter dans les îles $(16,82 \%)$ et au sud $(15,90 \%)$. Cependant, si l'on observe ce qui se passe dans les régions, la réalité apparaît beaucoup plus variée. Au nord, les régions occidentales donnent des chiffres très nettement supérieurs qui sont de l'ordre de $24 \%$ dans les classes du Piémont. Au sud, l'option pour le français atteint un maximum en Basilicate $(22,69 \%)$ et dans les Pouilles (19,14 \%). La demande apparait encore beaucoup plus diversifiée si l'on se réfère aux provinces et cela pour des raisons évidentes de contiguïté territoriale. Par exemple, à Impéria, 61,02 \% des classes s'orientent vers le français et à Turin, 28,65 \%.

\section{L'espagnol}

La demande pour l'espagnol est assez réduite puisqu'elle ne concerne que 1,47\% des classes. Complètement absente dans certaines zones (Vénétie, Trentin et Abruzzes), la langue espagnole est présente dans quarante-cinq provinces. Les taux les plus élevés se trouvent en Sardaigne (4,94\%), en Lombardie (3,99\%). La demande en faveur de la langue espagnole, bien qu'étant limitée, n'est pas concentrée dans des zones territoriales spécifiques, mais apparait disséminée sur l'ensemble du territoire. La demande la plus forte provient de Cagliari (8,77 \%) et de Milan (6,48 \%). 


\section{L'allemand}

23 très forte concentration dans le Trentin où elle provient de $94,57 \%$ des classes. Elle enregistre, en outre, un niveau relativement significatif dans la région Frioul-VénétieJulienne ( $7,19 \%$ des classes) parmi lesquelles il faut compter un pourcentage très élevé pour Trieste dans les écoles de langue Slovène (30,26 \%).

24 La stratégie de l'introduction de la langue étrangère suivie durant ces cinq premières années (1990-1995) s'est attachée à mettre en évidence la demande, à préparer une première mise en place de ressources professionnelles, à assurer le début d'un enseignement de la langue étrangère au niveau du CE2. Elle s'est intéressée, au niveau des écoles, d'un point de vue fonctionnel, aux modalités d'organisation et de programmation.

\section{NOTES}

1. Insegnamento lingua straniera scuole elementari (ILSSE).

2. Loi $n^{\circ} 148$ du 5 juin 1990.

\section{RÉSUMÉS}

L'enseignement des langues étrangères bénéficie de l'importante innovation apportée par la réforme concernant l'enseignement modulaire à l'école élémentaire. Pour l'enseignement précoce des langues, des cours de formation différenciés sont proposés aux enseignants, notamment dans le domaine de la linguistique, de la pédagogie et de l'interculturel.

\section{INDEX}

Index géographique : Italie

Mots-clés : école primaire, enseignement des langues, formation des enseignants, langues étrangères, enseignement précoce des langues vivantes 
AUTEUR

FRANCESCA BERTÈ

Professeur à l'Istituto Regionale di Ricerca di Sperimentazione e di Aggiornamento Educativo (IRRSAE) Lombardia, Milan, Italie. 